Bellori to begin with. At the same time, however, they are also full of useful information for anyone interested in seventeenth-century cultural politics and systems of patronage, or in the history of antiquarianism. Scholars interested in Bellori himself will find them especially useful in illuminating the relationships between Bellori's varied fields of activity and his complex relationships with patrons, collectors, artists, and theoreticians.

\title{
SHARON GREGORY
}

\section{St. Francis Xavier University}

\section{Scalabrini, Massimo. L'incamazione del macaronico. Percorsi nel comico folenghia- no. Bologna: Il Mulino, 2003. Pp. xix, 190. ISBN 88-15-09455-5. 15 Euro.}

"Phantasia mihi plus quam phantastica venit / historiam Baldi grassis cantare Camoenis," scrive Teofilo Folengo in apertura del Baldus (Torino: Einaudi, 1989, libro primo, vv. 1-2, 2), capolavoro rinascimentale che ancora attende di trasformarsi da spettrale a 'reale presenza' del canone letterario italiano. Di questa 'fantastica fantasia' si occupa l'originale, interessante e colto lavoro di Massimo Scalabrini, il cui coraggio intellettuale dovrà essere particolarmente sottolineato se si considera come abbia scritto questo lavoro su un aspetto importante dell' 'altra' tradizione italiana, quella che dai suoi esordi sino ad oggi sfida il dominio della retorica 'in lingua,' nell'ambito della accademia letteraria nordamericana, ancor oggi spesso sclerotizzata in stanchi e ripetitivi esami delle tre corone e dei più conosciuti e 'sicuri' autori del canone, quelli che con più certezza assicurano un lavoro universitario. L'ambizioso scopo del volume di Scalabrini è, come si legge nell'introduzione, di investigare "la teologia del comico" espressa dalla produzione letteraria di Folengo, che non si limiti al suo testo più conosciuto ma anzi lo metta in dialogo con le opere in italiano e in latino dello stesso scrittore (17). Scalabrini giustamente insiste come attraverso un esame di Folengo si possa determinare "la genealogia macaronica della moderna vocazione al 'misto'" (15). Lo studioso crede identificare "nell'evento incarnazionale (nell' "umanazione" del Verbo giovanneo) insieme il motivo e la matrice della poetica contaminatoria," poiché, secondo Scalabrini, "la poetica del macaronico folenghiano va posta in relazione con l'emergere e affermarsi, tra Quattrocento e Cinquecento, di un orientamento teologico teso a sottolineare l'incarnazione e l'umanità" di Cristo (16). Che l'incarnazione del Verbo possa essere la fonte originale della vocazione letteraria italiana al "misto" è ipotesi di grande interesse, anche se ulteriori analisi sarebbero necessarie per confermare o smentire se ne sia sempre stata e ne sia ancora il "motivo" formante. In ambito contemporaneo, ad esempio, un'indiscutibile connotazione religiosa è presente in Testori, scrittore che Scalabrini evita di citare, oltre che Pasolini, ma sarà più arduo da provare ad esempio in Fo. Ma varrà la pena ricordare come "Teofilo" sia il nome che Girolamo Folengo scelse quando nel 1509 pronunciò i voti nel convento benedettino di Sant'Eufemia, nei pressi di Brescia.

I capitoli di maggiore interesse del lavoro in questione sono il primo ed il secondo. Nel primo, dal titolo "Puer Macaronicus" (19-46), con grande sapienza e 
cultura l'autore porta alla superficie gli echi letterari (soprattutto numerosi cantari medievali, ma anche l'Orlandino, altro testo in italiano dello stesso Folengo) e soprattutto teologici presenti nella descrizione della nascita di Baldus, descritta nella seconda parte del secondo libro e nella prima del terzo, che apertamente echeggia la narrativa della natività. Rielaborando una dichiarazione di Bachtin concernente i canoni letterari dell'età classica, Scalabrini ricorda come "la scelta dell'infanzia e dei suoi referenti grotteschi ... equivale ... a una decisiva presa di posizione poetica," a dire, "la promozione di quanto è solitamente relegato nel dominio dell'incompiuto, dell'eccessivo e del facile alla sfera di ciò che domanda di essere espresso" (23-24). Pagine molto coinvolgenti sono dedicate al riso del neonato ("Ille nihil plorat, sed vultu grignat alegro") che l'autore vede connesse a delle riscritture della natività cristiana presenti in alcune leggende apocrife, oltre che alla quarta ecloga di Virgilio (Folenfo, Baldus, libro secondo, v. 470, 72) Il riso del Baldus neonato costituisce, secondo l'autore, "la scoperta centrale della poesia folenghiana dell'infanzia" ed è "il lascito forse più prezioso affidato dal grande macaronico alla tradizione (e dell'ermeneutica) del comico" (46).

Il secondo capitolo (47-82) esamina la figura di Cingar, compagno di Baldus, che l'autore legge alla luce delle sue fonti letterarie, soprattutto la figura topica di Margutte attraverso il Pulci, Boiardo e Ariosto, ma anche il Momus di Alberti. La centralità del personaggio di Cingar risiede, come lo studioso mette bene in risalto, nella sua natura emblematica di "reietto," dello "straniero privo di protezione" (67). Nel terzo capitolo dal titolo "Palpabili voci," Scalabrini pone il tema dell'infanzia presente nel Baldus in dialogo con altre opere di Folengo, ad iniziare dalla Palermitana ma includendo anche il successivo Umanità del Figliuolo di Dio. In questa parte, grazie a queste letture incrociate, Scalabrini prova una sua convincente intuizione riguardante la "vocazione del macaronico folenghiano." Lo studioso afferma che tale "vocazione" riguarda un "meditare sull'umanità pre-soggettiva e pre-linguistica dell' infans, il cui paradigma è naturalmente il Cristo bambino" (88). Scalabrini sintetizza la sua ricerca in questo campo affermando che "la poesia del mantovano dispiega un'attenzione sistematica all' 'Umanità del Figliuolo di Dio,' più volte ne contempla, in particolare, il momento della nascita" (107). Interessante, ma meno probante e necessario, è il seguente esame del concetto del "toccare con mano" (124) che l'autore allarga ad altri scrittori rinascimentali (le commedie di Ariosto, Machiavelli, Aretino, Della Porta e Giordano Bruno), esame che occupa una sezione del quarto capitolo e che sembra esulare dalla convincente analisi della poetica di Folengo (124-35).

In conclusione, il lavoro di Massimo Scalabrini si propone quale colto ed innovativo studio su un autore ed un fenomeno letterario di centrale importanza per la cultura italiana, non solo rinascimentale.

ARMANDO MAGGI

University of Chicago 